\title{
ASSOCIATION SOUS CONTRÔLE GRAMMATICAL : LE CAS DU A DU PLURIEL EXTERNE EN KABYLE
}

\author{
SAMIR BEN SI SAÏD \\ Faculté des Lettres; Laboratoire BCL \\ Université de Nice \\ 98, Bd Herriot ; B.P. 3209 \\ F-06204 Nice \\ France \\ bsamir76@gmail.com
}

\begin{abstract}
In Kabyle Berber there are two types of plural: one is internal and the other is external. The internal plural is characterized by the fact that the quality of the vowels changes, whereby the last vowel is always A, e.g., azarəz 'cord' $\rightarrow$ izuraz 'cords'. The external plural involves the suffixation of $-n$, e.g., i $\theta \beta i r$ 'pigeon' $\rightarrow i \theta \beta i r-n$ 'pigeons". Kabyle plural presents some melodic items which are not present in the singular form (e.g., iðmər 'chest' $\rightarrow$ iðmar- $n$ 'chests', iffəw 'horn' $\rightarrow$ i ifiw-n 'horns', aməçsa 'shepherd' $\rightarrow$ iməçsaw- $n$ 'shepherds'). Since they cannot be predicted, these items must be a piece of the root's lexical properties. For reasons to be determined, they do not appear in singular forms. It is shown that when a root of the external plural class bears an $\mathbf{A}$ that is not visible in the singular, this $\mathbf{A}$ is promoted to the surface in the plural form at any cost, i.e., even if damage on other items of the root is caused. As a consequence of the appearance of the $\mathbf{A}$, the gemination of a consonant or the expression of a vowel may be suspended, e.g., azəqqur 'log' $\rightarrow$ izəyran 'logs'. Yet, this A cannot be considered as a plural marker since it may well be absent from external plurals, e.g., argaz 'man' $\rightarrow$ irgaz- $n$ 'men'. Rather, it is an idiosyncratic property of the root, and the plural morpheme contains an explicit instruction for it to appear on the surface, involving, as it will be shown, a branching phenomenon. Thus, part of the plural morpheme consists in an instruction for a specific melodic item to branch.
\end{abstract}

Keywords: Kabyle Berber, internal plural, external plural, template, melodic items

\section{Introduction}

En kabyle, lors du passage du singulier au pluriel, hormis la marque du pluriel (le suffixe - $n$ pour le pluriel externe et la voyelle - $a$ en dernière 
position vocalique pour le pluriel interne), dans certains groupes de noms, on observe la présence de voyelles qui sont absentes au singulier.

Parmi les voyelles qui apparaissent seulement au pluriel, la voyelle A est particulière. Ce A dispose d'une priorité de se manifester au pluriel en dernière position vocalique. J'entends par «priorité» le fait que son association est obligatoire et se fait même au détriment d'autres voyelles et de géminées. Etant donné les restrictions gabaritiques que j'établirai, celles-ci sont perdues sous la pression de ce $\mathbf{A}$, qui pour autant n'est pas un morphème de pluriel puisqu'il y a des pluriels où il n'y apparaît pas. Le but de l'analyse est de montrer que ce $\mathbf{A}$ est une propriété lexicale des racines qui est «activée» au pluriel. En d'autres termes, l'association du A en question est sous contrôle grammatical. Il existe une situation typique d'un contrôle grammatical en arabe: la gémination de la consonne médiane dans une racine trilitère est un marqueur d'une catégorie grammaticale qui est l'intensif $\mathrm{C}_{1} \mathrm{~V}_{1} \mathrm{C}_{2} \mathrm{C}_{2} \mathrm{~V}_{2} \mathrm{C}_{3}$, i.e., $\mathrm{C}_{2}$ reçoit un ordre de s'associer.

En section 2, quelques aspects de la morpho-phonologie du kabyle. En section 3, je montrerai les caractéristiques du pluriel interne. La section $\mathbf{4}$, présente l'existence d'éléments mélodiques qui n'apparaissent qu'au pluriel. En section 5, je montrerai que $\mathbf{A}$ en dernière position vocalique est «prioritaire» au pluriel externe et en section $\mathbf{6}$, je présente l'analyse.

\section{Aspect morpho-phonologique du kabyle}

\subsection{Le pluriel en kabyle}

Il existe deux types de pluriel en kabyle: le pluriel externe (ou pluriel en -n), caractérisé par la suffixation de - $n$ à la forme du singulier (e.g., argaz $\rightarrow$ irgaz-n «homme»), le pluriel interne ou pluriel en A, caractérisé par le changement de timbre de certaines voyelles (apophonie) et l'apparition de la voyelle $\mathrm{A}$ en dernière position vocalique (azarəz $\rightarrow$ izuraz « corde ») (Chaker 1983).

Je tiens à signaler que j'ai relevé dans mon corpus 1475 pluriels externes sur 1960 contre 485 pluriels internes, ce qui montre la productivité du pluriel externe.

Notons que l'alternance de la voyelle initiale lors du passage du singulier au pluriel, i.e., a $\rightarrow$ i, est observable sur $97 \%$ des noms kabyles. Selon Basset (1945) cette voyelle ne fait pas partie de la racine et elle peut 
bien être un ancien article qui s'est figé et aujourd'hui, il est dépourvu de toute signification. Mais cette alternance ne joue aucun rôle dans la démonstration qui suit.

\subsection{Les voyelles du kabyle}

Le système vocalique du kabyle est composé de trois voyelles périphériques ${ }^{1}$ et d'un schwa, comme présenté sous (1):

(1) $\mathrm{i}$

u

a

D'après les grammaires traditionnelles (Chaker 1983 et Kossmann 1995), en kabyle, le schwa est inséré pour éviter une suite de trois consonnes (ou de deux consonnes en fin de mot) et il est prédictible, comme on peut le voir sous (2):

(2) (a) entre deux consonnes en finale de mot: C_C C : e.g., izəm « lion»

(b) entre $\mathrm{C}_{1}$ et $\mathrm{C}_{2}$ dans un groupe du type $\mathrm{C}_{1}-\mathrm{C}_{2} \mathrm{C}_{3} \mathrm{~V}$ : e.g., idəflan «neiges »

(c) entre une consonne et une géminée $\mathrm{C}-\mathrm{C}_{i} \mathrm{C}_{i}$ : e.g., azəqqur «bûche»

Enfin, notons que les séquences əCV et Cə\# sont illégales en kabyle : lorsqu'elles se présentent, le schwa n'est pas prononcé. Les séquences CC\#, $\mathrm{CCC}$, əCV et $\mathrm{Cə \#}$ sont donc interdites en kabyle.

\subsection{Le féminin en kabyle}

Il existe un morphème dit «féminin », caractérisé par l'affixation de $\theta$ à la forme du singulier, i.e., $\boldsymbol{\theta}-\sqrt{ } \mathbf{-} \boldsymbol{\theta}$, et qui couvre des valeurs sémantiques variées (Chaker 1998):

- sexe : mâle $\sim$ femelle = amyar « vieillard » $\sim \theta$-amyar- $\theta$ « vielle »

- taille : diminutif $\sim$ augmentatif $=$ asif "rivière » $\sim \theta$-asif- $\theta$ "petite rivière »

- inclusion : collectif $\sim$ singulatif $=$ awətt $^{\uparrow}$ uf $«$ les fourmis » $\sim \theta$-awətt ${ }^{\uparrow}$ uf- $\theta$ 《une fourmis »

- péjoratif : argaz « homme » $\theta$-argaz- $\theta$ « femmelette »

${ }^{1}$ J'entends par voyelles périphérique les voyelles extrêmes par opposition aux voyelles centrales qui n'existent pas en kabyle. 


\section{Le pluriel interne ou pluriel en -a}

L'appellation «pluriel interne» est due au fait qu'aucun élément externe à la racine ne s'ajoute à la forme du singulier, et le «pluriel en - $a$ » par rapport à la voyelle $a$ qui apparaît toujours en dernière position vocalique. Voici sous (3) des exemples de pluriel interne:

(3)

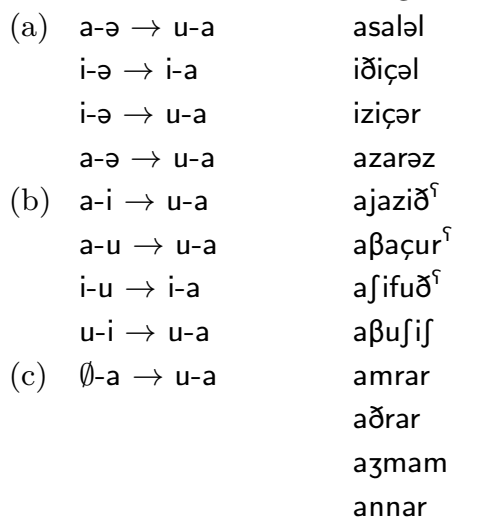

$\begin{array}{ll}\text { Pluriel } & \text { Glose } \\ \text { isulal } & \text { tuteur } \\ \text { iðiçal } & \text { creux de la main } \\ \text { izuçar } & \text { corde en sparte tressé } \\ \text { izuraz } & \text { morceau de corde } \\ \text { ijuzað์ } & \text { coq } \\ \text { ißuçar }^{\Im} & \text { figue précoce } \\ \text { ififað } & \text { sandales en peau de bœuf } \\ \text { ißufas } & \text { jeune frêne } \\ \text { imurar } & \text { corde } \\ \text { iðurar } & \text { montagne } \\ \text { izumam } & \text { mesure rase } \\ \text { inurar } & \text { aire à battre }\end{array}$

Sous (3), on observe l'invariable apparition d'un $\mathrm{A}$ entre $\mathrm{C}_{2}$ et $\mathrm{C}_{3}$, i.e., en tant que dernière voyelle et quelle que soit $\mathrm{V}_{3}$ au singulier $(ə, \mathrm{i}, \mathrm{u}, \mathrm{ou}, \mathrm{a})$. On peut donc considérer que ce $\mathrm{A}$ est le morphème du pluriel ou tout au moins une partie de ce marqueur.

\section{Absence vs. présence d'éléments mélodiques}

Les exemples sous (4) montrent que le pluriel externe en kabyle présente des voyelles et des consonnes qui sont absentes au singulier. Cela signifie que la forme sous-jacente des racines peut comporter des segments qu'on ne voit pas nécessairement au singulier. La démonstration de cet état de fait est l'objet de la présente section.

Les exemples sous (4) révèlent des éléments mélodiques qui font surface seulement au pluriel. Ces éléments ne sont pas prédictibles et seul le pluriel peut nous renseigner sur leur nature. On dira donc que ces éléments mélodiques sont présents dans le lexique et appartiennent à la racine mais, pour des raisons qui seront développées ci-dessous, ils n'apparaissent pas au singulier. 
(4)

\begin{tabular}{|c|c|c|c|}
\hline & Singulier & Pluriel & Glose \\
\hline & $u \iint \partial n$ & $u \iint a n-n$ & chacal \\
\hline & ¡ðmər & iðmar-n & côté de poitrine \\
\hline & 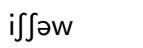 & a $\iint i w-n$ & corn \\
\hline & ifrəw & ifriw-n & épluchure \\
\hline & аßәqqа & aßəqqaj-n & gifle \\
\hline & ајuðu & ajuðuj-n & dépôt de détritus \\
\hline & ayənza & iуәnzaw-n & cuillère \\
\hline & aməçsa & iməçsaw-n & berger \\
\hline & $\theta$-iləf- $\theta$ & $\theta$-ilfa $\theta$-in & laie \\
\hline & $\theta$-alwəs- $\theta$ & $\theta$-iləwsa $\theta$-in & belle-soeur \\
\hline
\end{tabular}

\section{La priorité de A au pluriel externe}

J'ai classé les noms en fonction de leur forme au singulier. Le tableau suivant montre le résultat (qui pour l'instant est partiel).

\begin{tabular}{|c|c|c|}
\hline Classes & Formes & Exemples \\
\hline Class 2 & VCCC & 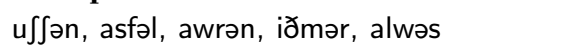 \\
\hline Class 3 & VCVC & amur, afus, agus, amud, asif \\
\hline Class 4 & VCVCVC & azuliy, aßaliy, ayanim, aßagus \\
\hline Class 5 & VCVCC & aqalə $\beta$, ißiqəs, azarəz, asawən, asuləf \\
\hline Class 6 & $\mathrm{VCC}_{\boldsymbol{i}} \mathrm{C}_{\boldsymbol{i}} \mathrm{VC}^{3}$ & 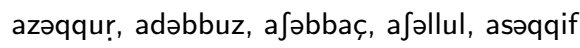 \\
\hline Class 7 & VCCCVC & aməhraz, igərgis, asəfru, akərfuf, aməçsa, \\
\hline Class 8 & VCCVC & argaz, uzzal, afrux, axxam, afzim, azniq \\
\hline
\end{tabular}

On voit sous (6) des exemples d'apparition de A au pluriel externe. En (a), des exemples d'une classe donnée où l'apparition de $\mathrm{A}$ au pluriel est accompagnée de la chute d'une autre voyelle. En (b), les noms de la même classe mais sans l'apparition du A.

Si le A était un exposant du marqueur de pluriel, on s'attendrait à ce qu'il soit présent dans tous les noms d'une même classe, ce qui n'est pas le cas. Le A en question ne peut pas être prédictible, il doit donc faire partie de l'information lexicale de la racine.

${ }^{2}$ On observe que la consonne $\theta$ apparaît seulement au féminin pluriel car elle a besoin d'une voyelle pleine à sa droite.

${ }^{3}$ La séquence $\mathrm{C}_{i} \mathrm{C}_{i}$ note une géminée. 
(6) Classe

\section{Processus internes}

(a) chute de $\mathrm{i}$

(b)

(a) chute de u

(b)

(a) chute de a chute de a, dégém. de $z$

(b)

6
(a) chute de $\mathrm{u}$, dégém. de $\mathrm{tt}^{\varsigma}$ chute de i, dégém. de qq

(b)

3

\begin{tabular}{|c|c|c|}
\hline Singulier & Pluriel & Glose \\
\hline$a_{j}{ }^{w} \lim$ & $i j^{w} ə l m a-n$ & peau \\
\hline azri $\beta$ & izərßa-n & ruelle \\
\hline afzim & ifzim-n & broche \\
\hline iffis & iffis-n & trèfle \\
\hline açsum & içəsma-n & viande \\
\hline amzur & iməzra-n & chevelure \\
\hline amðun & imðun-n & bassin \\
\hline aflus & iflus-n & gland séché \\
\hline afraj & ifərja-n & clôture \\
\hline uzzal & uzla-n & fer \\
\hline argaz & irgaz-n & homme \\
\hline axxam & ixxam-n & maison \\
\hline ajətt ${ }^{\uparrow} u m$ & ijəð'man & jeune pousse \\
\hline asəqqif & isəqfa-n & grande porte \\
\hline aləqqam & iləqqam-n & arbre greffé \\
\hline ifəddix & ifəddix-n & blessure \\
\hline iyil & iyall-n & bras \\
\hline afus & ifass-n & main \\
\hline amud & imudd-n & mesure de capacité \\
\hline
\end{tabular}

Le fait est alors qu'il n'apparaît pas au singulier, mais est systématiquement présent au pluriel. On a donc l'impression que ce A est en quelque sorte prioritaire au pluriel, mais peut être négligé au singulier.

Les exemples en (b) montrent que tous les noms ne possèdent pas un A sous-jacent: pas de voyelle A qui fait surface ni au singulier ni au pluriel.

\section{Analyse}

Dans cette section, l'association de A au pluriel externe donné en (6) peut-être expliquée par la structure gabaritique et l'hypothèse que les voyelles périphériques en kabyle sont sous-jacemment longues.

\subsection{Les gabarits}

Le gabarit, tel qu'il est conçu en afro-asiatique, est une séquence particulière de positions vocaliques et/ou consonantiques associée à une valeur morpho-sémantique. Le gabarit doit être satisfait, i.e., rempli, quel que 
soit le matériel vocalique et consonantique injecté dans la dérivation particulière. En arabe classique, il y a 15 formes verbales et chaque forme est contrainte par son gabarit. En (7), je donne quelques exemples de la Forme I au perfectif actif (McCarthy 1979; McCarthy-Prince 1999; Lowenstamm 2003).

$\begin{array}{lllll} & \text { Racine } & \text { Gabarit Forme I } & \text { Forme I } & \text { Glose } \\ \text { (a) } & \text { rsm } & \mathrm{C}_{1} \mathrm{VC}_{2} \mathrm{VC}_{3} & \text { rasam } & \text { dessiner } \\ & \mathrm{drs} & \mathrm{C}_{1} \mathrm{VC}_{2} \mathrm{VC}_{3} & \text { daras } & \text { étudier } \\ \text { (b) } & \mathrm{md} & \mathrm{C}_{1} \mathrm{VC}_{2} \mathrm{VC}_{3} & \text { madad } & \text { tirer } \\ & \mathrm{hb} & \mathrm{C}_{1} \mathrm{VC}_{2} \mathrm{VC}_{3} & \text { habab } & \text { aimer }\end{array}$

On voit sous (7a) qu'une racine saine (trilitère) à la Forme I, au perfectif actif, remplit le gabarit $\mathrm{C}_{1} \mathrm{VC}_{2} \mathrm{VC}_{3}$. Lorsque la racine est bilitère comme sous (7b), la deuxième consonne se propage sur la position $\mathrm{C}_{3}$ pour satisfaire le gabarit, car celui-ci comporte trois positions consonantiques.

\subsection{La longueur vocalique}

J'adopte ici l'hypothèse selon laquelle les voyelles périphériques en kabyle sont sous-jacemment longues. Dans cette perspective, le schwa est soit la réalisation d'un noyau vide non gouverné, soit une primitive mélodique I/U/A associée à une seule position vocalique.

(8)<smiles>[Y][Al][123I]</smiles>

$\mathrm{C}$<smiles>[Tl]I([Tl])[Tl]</smiles>

C<smiles>[V][Al][Tl]</smiles>

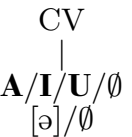

Cette hypothèse a été formulée au départ par Lowenstamm (1991) pour l'éthio-sémitique et l'arabe maghrébin, elle a été ensuite appliquée au système vocalique du kabyle par Bendjaballah (1995; 1999 ; et passim).

\subsection{Apparaître ou ne pas apparaître}

Je propose dans Ben Si Saïd (2009) que le gabarit du pluriel est constitué d'une séquence de 7 unités [CV], et que le singulier n'est pas gabaritique dans le sens où il n'impose pas une taille unique à tous les noms. En revanche, chaque classe de noms possède son propre gabarit: A titre d'exemple, j'illustre trois classes ci-dessous 
- Class 2 : e.g., argaz: VCCVC: $5 \mathrm{CV}$

- Class 6 : e.g., azəqqur: $\mathrm{VCC}_{i} \mathrm{C}_{i} \mathrm{VC}: 8 \mathrm{CV}$

- Class 3: e.g., asif : VCVC: 5 CV

Les voyelles qui font surface seulement au pluriel (cf. (4)) sont présentes dans la mélodie mais elles n'apparaissent pas au singulier parce que le gabarit du singulier ne leur offre pas assez d'espace pour brancher sur deux positions vocaliques : elles ne sont associées qu'à une seule position, ce qui les réduit à schwa ou zéro

Aussi, les consonnes qui font surface seulement au pluriel (cf. (4)) sont aussi présentes dans la mélodie au singulier. Si elles n'y apparaissent pas, c'est parce que le gabarit du singulier ne leur offre pas l'espace nécessaire pour qu'elles s'associent, et elles demeurent alors flottantes.

\subsection{L'association du A du pluriel externe}

Voici sous (9) l'analyse de quelques exemples donnés en (6a). Lorsque A est présent dans la spécification lexicale d'une racine, il est traité comme tous les autres éléments mélodiques au singulier où il peut n'être associé qu'à une seule position, ce qui le réduit en surface à schwa ou à zéro (cas sous 9a). Au pluriel en revanche, il reçoit l'ordre de s'associer sur deux positions et apparaît en tant que [a] en surface. Le gabarit du pluriel n'étant pas extensible, cette association «forcée» peut alors avoir des conséquences sur d'autres éléments de la racine, dont l'association peut être réduite à une seule position. Sous $(9 \mathrm{a})$ et $(9 \mathrm{~b})$, une voyelle qui est pleine au singulier s'en trouve ainsi réduite à schwa/zéro, et sous $(9 \mathrm{c}-\mathrm{e})$ cet effet est accompagné d'une dégémination, toujours en raison de la limitation d'espace imposée par le gabarit.

(9) (a) association de $\mathbf{A}$ et chute de $\mathbf{I}^{4}$

Singulier : [azniq]

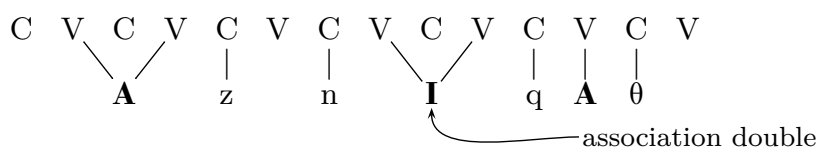

\footnotetext{
${ }^{4}$ Les voyelles qui chutent en surface restent, sous-jacemment, associées à une seule position vocalique.
} 
Pluriel : [izənqan]

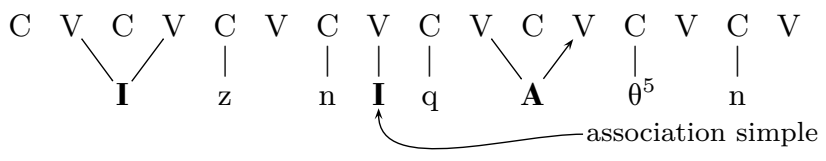

(b) association de $\mathbf{A}$ et chute de $\mathbf{U}$ :

Singulier : [açsum]

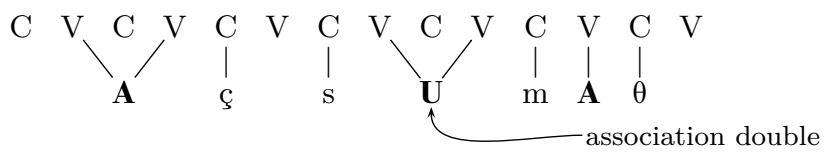

Pluriel : [içəsman]

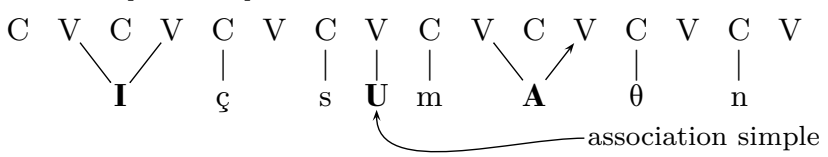

(c) association de $\mathbf{A}$, chute de $\mathbf{A}_{1}$ et dégémenination de $\mathrm{C}_{1}:^{6}$

Singulier : [uzzal]

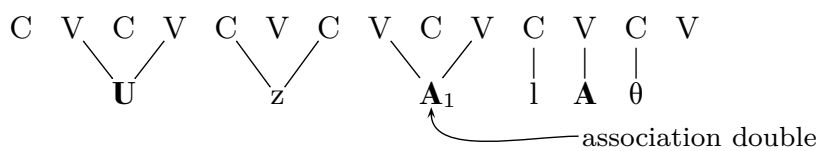

Pluriel : [uzlan]

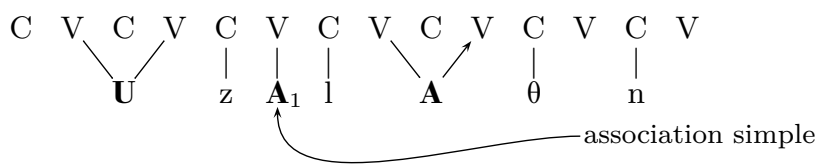

(d) association de $\mathbf{A}$, chute de $\mathbf{U}$ et dégémination de $\mathrm{C}_{2}$ :

Singulier : [ajətt $\left.{ }^{\complement} \mathrm{um}\right]$

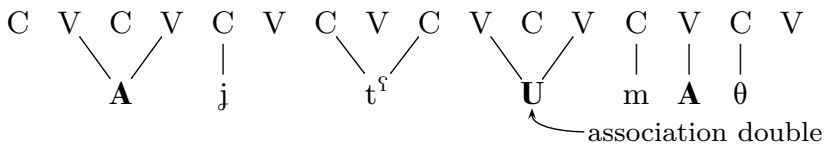

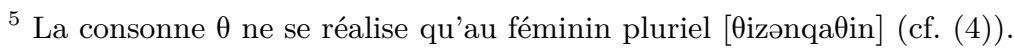

${ }^{6}$ Dans cet exemple, il y a une solution alternative qui est uzz-ə-lan, mais comme la langue ne veut absolument pas de [əCV], donc le schwa doit sauter, ce qui a comme effet la dégémination. 
Pluriel : [ijəð⿱一土man]

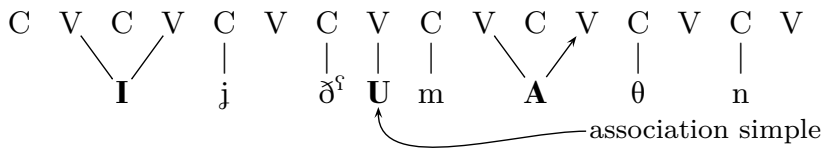

(e) association de $\mathbf{A}$, chute de $\mathbf{U}$ et gémination de $\mathrm{C}_{2}$ :

Singulier : [afus]

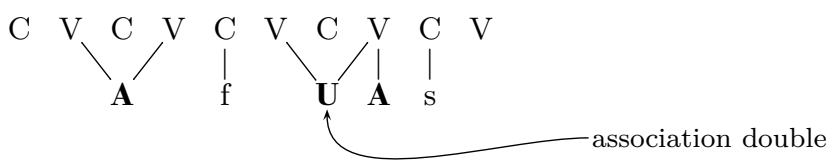

Pluriel : [ifassən]

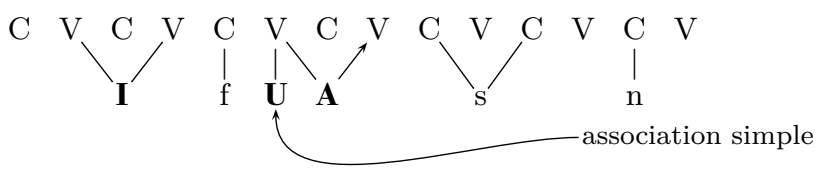

En (9.5) au singulier le $\mathbf{U}$ est associé à deux positions vocaliques et le A n'a pas assez d'espace pour brancher sur deux positions, ce qui donne la réalisation de surface [afus]. Au pluriel, comme A est prioritaire, il branche sur deux positions vocaliques et réduit le $\mathbf{U}$. Enfin, le $s$ gémine pour remplir le gabarit de $7 \mathrm{CV}$.

\section{Conclusion}

Les exemples ci-dessus montrent que le $\mathbf{A}$ qui apparaît en dernière position vocalique au pluriel externe fait partie de la racine; par ailleurs, il est appelé à s'associer à deux positions coûte que coûte au pluriel, ce qui peut entraîner la désassociation d'autres voyelles et/ou consonnes qui seront alors réduites à schwa/zéro ou à des consonnes simples. En d'autres termes, le $\mathbf{A}$ est lexicalement spécifié en tant qu'objet qui doit apparaître en surface au pluriel.

On peut supposer qu'en diachronie le pluriel était seulement interne, mais ensuite on a ajouté le pluriel externe qui a dysfonctionnalisé le morphème original, i.e., le $\mathbf{A}$ en dernière position vocalique. Celui-ci ou bien a fusionné avec le matériel mélodique de la racine et est devenu un ingrédient lexical, ou bien a été perdu. Dans le premier cas, la relation du A au pluriel est passée d'un exposant classique à une relation d'activation : 
une pièce spécifique de la mélodie d'une racine est lexicalement marquée pour être activée lorsqu'un autre morphème est concaténé.

L'association de $\mathbf{A}$ au pluriel est donc sous contrôle grammatical, comme le cas des formes I (intensif) et III (réciproque) en arabe classique (et plus généralement en sémitique) : la forme II donne l'ordre à $\mathrm{C}_{2}$ de géminer, et la forme III à $\mathrm{V}_{1}$ de s'allonger.

Guerssel et Lowenstamm (1990) ont identifié un gabarit commun aux formes verbales de l'arabe classique, avec un site [CV] (dit syllable dérivationnelle) qui accueille des opérations morphologiques et sur lequel $\mathrm{C}_{2}$ se propage à la Forme II et $\mathrm{V}_{1}$ à la Forme III, comme on peut le voir en (10) ci-dessous :

(10) $\sqrt{ } \mathrm{ktb}=$ écrire

Forme I

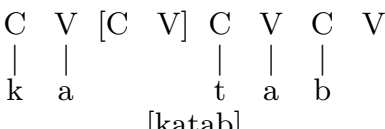
[katab]

Forme I

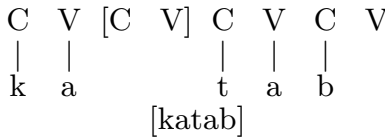

Forme II

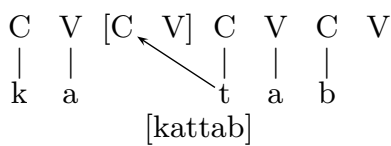

Forme III

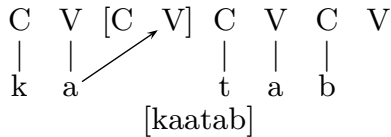

\section{Références bibliographiques}

Basset, André 1945. Sur la voyelle initiale en berbère. In : Revue Africaine 86 : 82-88.

Ben Si Saïd, Samir 2009. La formation du pluriel en kabyle. Mémoire de master 2, Université de Nice.

Bendjaballah, Sabrina 1995. Aspect du système verbal berbère (kabyle). Mémoire de DEA, Université de Paris 7.

Bendjaballah, Sabrina 1999. Trois figures de la structure interne des gabarits: activité morphologique du niveau squelettal des représentations phonologiques en berbère, somali et béja. Thèse de doctorat, Université de Paris 7 .

Chaker, Salem 1983. Un parler berbère d'algérie (syntaxe). Publications de l'Université de Provence, Aix-en-Provence.

Chaker, Salem 1998. Genre grammatical (masculin/féminin). In : Encyclopédie Berbère 20 : 3042-3045.

Guerssel, Mohamed-Jean Lowenstamm 1990. The derivational morphology of the Classical Arabic verb. Ms. Université Paris 7 \& UQAM. 
Kossmann, Maarten G. 1995. Le schwa en berbère. In : Journal of Africain Languages and Linguistics $16: 71-82$.

Lowenstamm, Jean 1991. Vocalic length and syllable structure in Semitic. In: Alain S. Kaye (ed.) : Semitic studies in honor of Wolf Leslau on the occasion of his 85th birthday, 949-65. Harrassowitz, Wiesbaden.

Lowenstamm, Jean 2003. A propos des gabarits. In: Recherches Linguistiques de Vincennes $32: 7-30$.

McCarthy, John J. 1979. Formal problems in Semitic phonology and morphology. Doctoral dissertation, MIT.

McCarthy, John J.-Alan S. Prince 1999. Prosodic morphology. In: John A. Goldsmith (ed.) : Phonological theory. The essential readings, 238-288. Blackwell, Malden MA \& Oxford. 